\title{
Développement des vertébrés: pas de notochorde en l'absence du facteur de transcription HNF3ß
}

Des gènes qui pourraient participer au développement précoce des vertébrés ont été identifiés. Plusieurs facteurs de transcription sont ainsi exprimés dans le centre organisateur et pourraient être impliqués dans ses fonctions au cours de la gastrulation $[1,2]$ : induction et régionalisation du système nerveux, dorsalisation du mésoderme... Le gène gsc (goosecoid) en est un exemple spectaculaire : s'il est exprimé de manière ectopique dans l'embryon de xénope, gsc provoque une duplication de l'embryon analogue aux célèbres duplications obtenues lors de greffes du centre organisateur par Spemann au début du siècle. Mais la preuve formelle de la participation d'un gène tel que gsc à ces processus passe par l'obtention de mutants de ce gène.

HNF3ß est un autre exemple de facteur de transcription exprimé dans le centre organisateur et, afin d'examiner son rôle au cours du développement, des souris dépourvues du gène HNF3ß (dites HNF3-/-) ont été obtenues par recombinaison homologue par les groupes de Janet Rossant à Toronto (Canada) et James Darnell et Tom Jessel à New York (USA) [3, 4]. A l'état homozygote, une létalité embryonnaire est observée. A la naissance, aucun embryon HNF3B-/n'est détecté, tous les embryons mutants ayant été résorbés; la létalité embryonnaire est donc précoce. Comment les embryons mutants sont-ils affectés?

HNF3ß a été identifié pour sa participation à la transcription spécifique de tissu de gènes hépatiques (d'où son nom, hepatic nuclear factor $3 \beta$ ) mais son expression dans plusieurs qu'il pouvait également jouer un rôle au cours de l'embryogenèse [5]. L'expression du gène HNF3ß est en effet activée peu après le début de la gastrulation dans une région où apparaît le nœud (l'équivalent chez la souris du centre organisateur des amphibiens), région située juste antérieurement au sillon primitif; puis, au cours de la gastrulation, le gène HNF3ß est transcrit dans le nœud et dans des tissus axiaux qui en émergent, la notochorde et l'endoderme définitif. Au cours de la neurulation, il est également activé dans la partie ventrale du tube nerveux avec laquelle la notochorde est en contact. C'est donc dans ces cellules que se manifeste le défaut primaire des souris HNF3ß-/-, l'absence de HNF3ß.

Le phénotype des embryons de souris HNF3ß-/- a été décrit par les deux groupes en conjuguant une analyse morphologique et une étude des domaines d'expression de gènes démarquant des territoires embryonnaires d'intérêt. A 8 jours et demi post coitum, l'embryon de souris normal possède la morphologie caractéristique des vertébrés, un cerveau divisé antéro-postérieurement en plusieurs domaines, comportant yeux, vésicules auditives et arcs branchiaux, et un tronc au mésoderme paraxial segmenté antéro-postérieurement en somites situés de part et d'autre de la notochorde. Chez les embryons de souris HNF3B-/-, de nombreuses structures embryonnaires sont affectées, aussi bien celles qui expriment normalement HNF3ß que d'autres. Dans un groupe d'embryons, aucune structure embryonnaire n'est reconnaissable; dans le second groupe d'embryons, les structures cépha- liques sont partiellement tronquées ; il est possible de reconnaître un tronc dépourvu de notochorde et à l'organisation dorso-ventrale bouleversée. Le tube nerveux ne présente pas de différenciation ventrale (plancher et motoneurones sont absents) et les somites sont peu développés. Les défauts dans les structures qui n'expriment pas le gène $H N F 3 \beta$ s'expliquent par l'absence de la notochorde. En effet, la participation de la notochorde à l'organisation dorsoventrale du tube nerveux et des somites est bien établie grâce aux travaux d'embryologie par greffes/ ablations [6]. A 8 jours $1 / 2$ post coitum, le phénotype le moins sévère consiste donc, dans le tronc, en un défaut primaire, l'absence de notochorde, et des défauts secondaires à cette absence. De manière analogue, dans le cerveau, l'absence de mésoderme axial pourrait être responsable des malformations observées. Quelle est donc alors l'origine de l'absence de notochorde chez les souris $H N F 3 \beta$-/-?

Cette structure caractéristique des vertébrés et des procordés comme les amphioxus, nos cousins invertébrés les plus proches $\left(\mathrm{m} / \mathrm{s} n^{\circ} 11\right.$, vol. 10 , p. 1178), émerge du nœud lors de la gastrulation puis disparaît ultérieurement pour laisser place à la colonne vertébrale. Chez les embryons $H N F 3 \beta-/$-, la notochorde n'apparaît jamais. Le facteur HNF3ß semble donc être un facteur de différenciation de la notochorde. Toutefois, dès le début de la gastrulation, les embryons dépourvus de HNF3ß sont affectés et ces défauts initiaux pourraient aussi bien être indirectement responsables de l'absence de noto- 
chorde : l'embryon, de taille réduite, possède un sillon primitif anormalement court et le nœud, d'où émerge normalement la notochorde, ne peut être identifié morphologiquement. L'expression de gènes marqueurs du nœud est également affectée. Le gène gsc est transcrit mais dans un domaine qui n'est plus circonscrit à l'ébauche du nœud comme chez les embryons normaux. Et le gène HNF $3 \beta$ lui-même n'est plus transcrit que de manière ectopique (son expression a pu être examinée chez les souris $H N F 3 \beta-/$ - par le groupe de $J$. Darnell qui, lors de l'inactivation du gène, a introduit l'ADNc de la Bgalactosidase en lieu et place de la séquence codante de $H N F 3 \beta$ ). En dépit de ces anomalies du noud, les cellules présentes semblent remplir certaines de ses fonctions ; ainsi, l'endoderme définitif, qui, comme la notochorde, dérive du nœud, apparaît normalement chez les embryons mutants. En définitive, HNF3ß est donc nécessaire à la formation du nœud et en particulier, au sein des cellules du nœud, à la spécification de la notochorde.

Les modèles rendant compte de l'embryogenèse précoce des vertébrés sont fondés sur la description des mouvements cellulaires par analyse clonale et font intervenir comme principal moteur du développement une cascade d'interactions entre tissus embryonnaires découvertes lors des études expérimentales chez les amphibiens et les oiseaux. L'étude fine des embryons dépourvus de HNF3ß au moyen des approches classiques de l'analyse génétique du développement (étude de mosaïques génétiques, étude de doubles-mutants...) permettra de mieux comprendre les processus en jeu au début de la gastrulation, la nature et les interactions des différentes cellules du nœud qui sont responsables de l'apparition des formes chez les vertébrés. Enfin pour ce qui est des fonctions de HNF3ß après la gastrulation, par exemple dans l'établissement/maintien de la polarité dorsoventrale du tube nerveux par la notochorde ou dans la différenciation hépatique, il faudra attendre le développement d'outils d'inactivation génique conditionnelle...[7]

J.-P.C.

1. Condamine H. L'organisateur de l'embryon d'amphibien. médecine/sciences $1992 ; 8: 483-6$.

2. Joly JS, Cohen-Tannoud ji M. I'unite de la gastrulation chez les vertébrés. méderine/sriences 1994 ; 10): 84-90.

3. Ang SL, Rossant J. HNF3B is essential for node and notochord formation in mouse development. Cell $1994 ; 78: 561-74$.

4. Weinstein D, Ruiz i Altaba A, Chen W, Hoodless $P$, Prezisos V, Jessel T, Darnell J. The wingedhelix transcription factor HNF3B is required for notochord developement in the mouse embryo. (isll $1994 ; 78: 575-88$.

5. Ang SI., Wierda A, Wong I), Stevens $K$, (Cascio) $S$, Rossant J, Zaret $K$. The formation and maintenance of the definitive endoderm lineage in the mouse : involvement of $\mathrm{HNF} 3 /$ forkhead related protcins. I)evelopement 1993 ; 119 : 1301-15.

6. Concordet JP. Morphogenèse, acide rétinoïque et Sonic Hedgehog. médecine/sciences $1994 ; 10: 57(1-3$. 7. Viville $S$. Recombinaison homologue : nouveaux vecteurs, nouvelles perspectives. méderine/ sciences 1995 (sous presse).

\section{BRÈVES}

Insuffisance rénale chronique et protéine inhibitrice des fonctions granulocytaires (GIP) : un facteur de dysrégulation de l'immunité locale sur-synthétisé par les cellules mésangiales. L'inflammation glomérulaire est associée à une expression excessive de certains facteurs locaux tels l'IL6, I'IL8 et des protéines de la matrice extracellulaire. En fait, une part essentielle du contrôle de la lésion glomérulaire s'effectue par les cellules mésangiales qui ont la propriété de synthétiser et de répondre à des médiateurs réglant la mise en jeu des réactions vasculaires ou inflammatoires. Il en est ainsi de l'IL6, de l'IL8 et d'un nouveau facteur, la protéine inhibitrice des fonctions granulocytaires ou granulocyte inhibitory protein (GIP) [1]. Cette protéine GIP de $23 \mathrm{kDa}$, initialement isolée à partir d'un ultrafiltrat d'urines de patients atteints d'insuffisance rénale, inhibe le chimiotactisme et l'activité bactéricide des granulocytes. La présence de cette protéine en quantité abondante dans l'insuffisance rénale pourrait expliquer la susceptibilité de ces malades aux infections. De plus, cette GIP a des fonctions de régulation immunitaire en stimulant l'expression autocrine de l'IL6 et de l'IL8 par les cellules mésangiales [2]. Ainsi, des concentrations nanomolaires de GIP stimulent spécifiquement la synthèse du facteur API en activant la transcription à la fois de $c$-jun et de $c$-fos. Fait intéressant, la présence simultanée de GIP et d'IL6 aboutit à la stimulation synergique des deux facteurs de transcription. Ainsi, la présence de GIP au cours de l'insuffisance rénale pourrait favoriser une réponse exagérée des cellules mésangiales à divers médiateurs pro-inflammatoires en activant la synthèse locale de cytokines telles que l'IL6 et l'IL8. Cette protéine, une fois son gène cloné, pourrait représenter un agent pharmacologique utile dans certaines conditions pathologiques.

[1. Horl WH, et al. Proc Natl Acad Sci USA $1990 ; 87$ : 6353-7.]

[2. Ziesche R, et al. Proc Natl Acad Sci USA 1994 ; 91 : 301-5.]
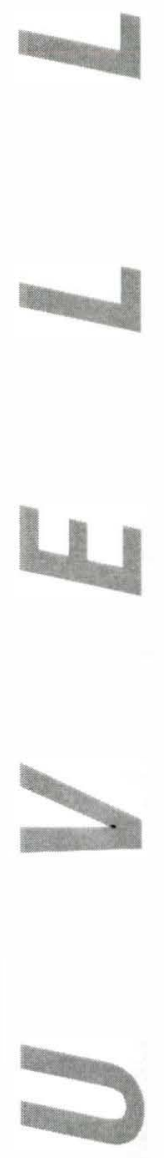

$m / s n^{\circ} 1$, vol. 11 , janvier 95 
Implication de la famille des MAP kinases dans le contrôle de l'osmolarité cellulaire. Le maintien de l'homéostasie cellulaire est une propriété fondamentale des cellules de mammifères. Ainsi, l'exposition des cellules à un changement de condition osmotique déclenche une réponse immédiate qui vient rétablir le volume cellulaire. Elle implique une modification des transports ioniques membranaires et l'accumulation de solutés non polaires dans la cellule, mais les mécanismes de signalisation responsables de cette réponse demeurent inconnus. Cependant, deux études récentes suggèrent que des membres de la famille des MAP (mitogen-activated protein) kinases sont impliqués dans le contrôle de l'osmolarité cellulaire [1, 2]. Dans une première étude, l'équipe de Davis (Boston, MA, USA) montre que l'exposition de cellules $\mathrm{CHO}$ à un milieu hyperosmolaire stimule l'activité enzymatique de Jnkl, un cousin éloigné des MAP kinases Erkl et Erk2 [1]. Cette activation est abolie par la mutation des sites de régulation $\mathrm{Thr}^{183}$ et $\mathrm{Tyr}^{185}$ en amont du sousdomaine VIII, démontrant ainsi que la phosphorylation de l'enzyme est nécessaire à son activité. Une telle voie de signalisation a également été décrite chez la levure $S$. cerevisiae. Cette levure répond à une augmentation de l'osmolarité extracellulaire par une production accrue de glycérol. L'analyse génétique de cette réponse a révélé l'implication du gène $H O G 1$, qui code pour un membre de la famille des MAP kinases [3]. Les mêmes travaux du groupe de Davis démontrent que l'expression de Jnkl dans une souche de levure dépourvie du gène $H O G 1$ complémente le défaut de croissance des cellules en milieu hyperosmolaire. En revanche, aucune complémentation n'est observée en présence d'un mutant de Jnkl dépourvu d'activité enzymatique ou d'un mutant dont les sites de phosphorylation sont défectueux. Dans une seconde étude, Han et al. (la Jolla, CA, USA) rapportent l'isole- ment d'un clone d'ADNc de souris codant pour un nouveau membre de la famille des MAP kinases. L'analyse de la séquence de cette nouvelle protéine, nommée p38, révèle une analogie particulièrement importante avec la protéine kinase de levure HOG;. D'une manière similaire à Jnkl, la synthèse de p38 est capable de complémenter une mutation de $H() ; 1$ dans $S$. cerevisiae. De plus, les auteurs montrent que l'exposition de monocytes ou de lymphocytes B à un milieu hyperosmolaire induit la phosphorylation en tyrosine de p38. L'ensemble de ces travaux implique les protéine kinases Jnkl et p38 dans une voie de signalisation pour le contrôle de la réponse des cellules de mammifères aux changements de pression osmotique. Ces enzymes pourraient jouer un rôle direct en modulant la fonction de transporteurs membranaires d'ions ou de solutés non polaires. De façon alternative, ces membres de la famille des MAP kinases pourraient régler l'activité de facteurs de transcription qui contrôlent l'expression des gènes de ces transporteurs membranaires.

[1. Galcheva-(jargova Z, et al. Science 1994 ; 265 : 806-8.]

[2. Han J, et al. Science 1994; 265: 808-11.]

[3. Brewster JL, et al. Science 1993; 259 : 1760-3.]

La thérapie génique dans le traitement des resténoses artérielles a fait ses preuves chez le porc. La survenue d'un traumatisme de la paroi artérielle induit la synthèse de produits de gènes qui stimulent la migration et la prolifération des cellules du muscle lisse, conduisant à l'hyperplasie de l'intima. Ce phénomène contribue à la pathogénie de plusieurs désordres cardiovasculaires dont l'athérosclérose. Un tel traumatisme peut être aussi iatrogène, par exemple, au cours d'une dilatation par angioplastie d'une artère coronaire sténosée. Dans ce cas, la prolifération cellulaire entraîne une resténose particulièrement réfractaire aux traitements conventionnels. Des approches moléculaires pour limiter ces proliférations à des sites spécifiques au niveau artériel pourraient permettre une meilleure compréhension de la pathogénie, voire constituer une voie thérapeutique. L'équipe de Gary et Elizabeth Nabel à l'Université du Michigan à Ann Arbor (USA) vient de publier des résultats convaincants, basés sur l'utilisation de la thérapie génique [1]. Ils ont travaillé sur le porc, car le lit artériel du porc a une taille et une structure qui sont similaires aux artères coronaires humaines dans leur biochimie et leur histologie. Ce groupe a montré que le traitement par un gène du virus Herpès codant pour l'enzyme thymidine kinase (tk), combiné à un traitement par l'antiviral ganciclovir, bloquait la prolifération des cellules du muscle lisse consécutive au traumatisme dû au ballon d'angioplastie, au niveau des artères ilio-fémorales du porc. Dans un premier temps, les artères ont été soumises à un traumatisme, soit de une minute, soit de cinq minutes. Puis, dans un deuxième temps, (quelques minutes après), le gène $t k$, contenu dans un adénovirus servant de vecteur, fut introduit dans les artères par un cathéter. Enfin, dans un troisième temps (36 heures après), les porcs furent traités pendant six jours par du ganciclovir $50 \mathrm{mg} / \mathrm{j}$ par kilogramme de poids. Trois semaines après le traumatisme et l'infection par l'adénovirus, la détermination du rapport intima/media par morphométrie quantitative montra une diminution significative de ce rapport de $87 \%$ pour les traumatismes de une minute, et de $54 \%$ à $59 \%$ pour les traumatismes de cinq minutes chez les animaux traités par rapport aux animaux non traités. Ces résultats restèrent stables six semaines après le traumatisme. Aucune toxicité ne fut retrouvée. L'enzyme tk n'agit pas directement sur la prolifération des cellules mais les rend susceptibles au ganciclovir, un analogue de nu- 
cléotide. Ce médicament, qui est d'ailleurs inoffensif par lui-même, est phosphorylé par l'enzyme tk, puis son incorporation dans l'ADN induit une terminaison de chaine chez les cellules en division, entraînant la mort cellulaire. Ces résultats sont d'autant plus encourageants qu'une autre équipe au National Heart Lung and Blood Institute (USA) a appliqué une stratégie identique au niveau des artères carotides du rat et retrouve, che $z$ les animaux traités, une diminution de la prolifération artérielle. Plusieurs problèmes restent toutefois à résoudre avant de pouvoir envisager une telle approche che $z$ l'homme. Tout d'abord, les artères utilisées dans ces travaux sont saines et l'on peut imaginer que ce traitement pourrait ne pas être efficace sur des artères athéroscléreuses. Ensuite, pour la délivrance de l'adénovirus au niveau de la plaie artérielle, le flux sanguin doit être bloqué $20 \mathrm{mi}$ nutes, ce qu'une artère fémorale peut subir, mais pas une coronaire. Enfin, l'innocuité d'une telle approche n'est pas prouvée. L'avenir réside peut-être dans la délivrance dans l'artère d'un cocktail de gènes agissant sur différents aspects de la resténose. A titre d'exemple, un autre gène candidat est celui codant pour l'enzyme oxyde nitrique synthétase dont le produit, l'oxyde nitrique, est vasodilatateur et prévient la formation du caillot [2].

[1. Ohno T, et al. Science 1994; 265 : 781-4.]

[2. Barinaga M. Science 1994; 265 : 738.]

Des mutations du gène $N F-H$ des neurofilaments qui prédisposent à la sclérose latérale amyotrophique. Les neurofilaments, un des principaux éléments du cytosquelette che $z$ les neurones, sont assemblés par la polymérisation de trois protéines appelées NF-L, NF-M et NF-H. Des accumulations anormales de neurofilaments dans les motoneurones constituent une des caractéristiques pathologiques de la sclérose latérale amyotrophique (SIA). Pour la première fois en 1993, des études sur des souris transgéniques ont fourni l'indication que les neurofilaments pouvaient contribuer à la pathogénie de la SLA [1, 2], notamment en affectant le transport axonal. Cette hypothèse est maintenant confirmée par la découverte de mutations du gène $N F-H$ des neurofilaments chez certains malades atteints de la SIA [3]. En effet, des chercheurs de l'université Mc(jill à Montréal, en collaboration avec le (entre SLA de l'Hôtel-Dieu de Paris, rapportent la perte de codons dans la région du gène $N F-H$ contenant les motifs répétés de la phosphorylation chez 5 des 356 cas de SLA étudiés. Aucune mutation n'a été identifée chez 306 individus normaux. Par quel mécanisme ces mutations du gène $\mathrm{NF}$-H peuvent-elles causer la SLA ? Les auteurs proposent que ces mutations pourraient sensiblement modifier les propriétés d'interactions de la protéine NF-H, ce qui prédisposerait les neurofilaments à s'accumuler de façon anormale et à bloquer le transport axonal.

[1. Côté F, el al. Ciell 1993 ; 73 : 3546.]

[2. Xu Z, el al. Cell $1993 ; 73$ : 2333.]

[3. Figlewicz DA, et al. Hum Mol Genet $1994 ; 3$ : 1757-61.]

- Thérapie génique des maladies dominantes: l'exemple de la drépanocytose. La thérapie génique classique consiste à apporter un gène qui, dans les maladies génétiques, compensera le déficit fonctionnel du gène homologue muté. Cette stratégie peut bien s'appliquer aux maladies par "perte de fonction ", dans lesquelles l'anomalie est secondaire à une insuffisance fonctionnelle. En revanche, le transfert d'un gène normal peut ne pas suffire dans le cas de maladies avec "gain de fonction" dans lesquelles les signes pathologiques sont la conséquence d'une fonction anormale, et non pas d'une insuffisance de fonction; ces maladies avec "gain de fonction" sont pratiquement toujours dominantes puisqu'une copie d'un gène codant pour une protéine délétère suffit à l'apparition des symptômes. Dans ces cas, une stratégie possible de traitement serait l'inactivation du gène codant pour ces protéines mutantes, par exemple par une stratégie anti-sens ou anti-gène [1]. Une autre stratégie serait de transférer un gène codant pour une protéine capable de contrecarrer les effets délétères du produit du gène muté. Cette approche est bien illustrée par un récent article de McCune et al. (Birmingham AL, Philadelphie, PA, USA). Ces auteurs se sont fixé pour objectif de créer une chaîne de globine capable d'éviter la précipitation de l'hémoglobine S caractéristique de la drépanocytose. La mutation drépanocytaire consiste dans le remplacement d'un acide glutamique (c'est-à-dire un acide aminé polaire) par une valine (acide aminé hydrophobe) en position 6 de la chaîne de globine $\beta$. A faible pression en oxygène, la valine $\beta 6$ interagit avec une poche hydrophobe naturelle présente à la surface d'un second tétramère d'hémoglobine, ce qui entraîne une polymérisation de l'hémoglobine $\mathrm{S}$ en de longues fibres composées de 7 paires de doubles chaînes de polymères. Ces fibres réduisent la déformabilité des érythrocytes, leur faisant prendre leur forme caractéristique en faux (anémie falciforme) et conduit à l'occlusion des petits capillaires. Certaines hémoglobines sont moins sensibles que l'hémoglobine adulte normale (HbAl) à la polymérisation induite par la présence d'HbS. Ainsi en est-il de l'hémoglobine foetale (c'est-à-dire un tétramère $\alpha 2 \gamma 2)$. Mc Cune el al. ont synthétisé par génie génétique un gène codant pour une chaîne $\beta$ humaine mutée, différant de la chaîne $\beta$ normale en deux positions : remplacement de l'alanine 22 par un acide 
glutamique et de la thréonine 87 par une glutamine. Ce gène ainsi modifié code pour une chaîne $\beta$ AS (anti-sickling). Des souris transgéniques ont alors été créées portant des transgènes humains pour la chaîne $\alpha$ et la chaîne $\beta$ AS, sous le contrôle des séquences régulatrices assurant une très forte expression de tels transgènes (LCR, locus control regions, $m / s n^{\circ} 3$, vol. 6 , p. 314, $n^{\circ} 1$, vol. 8, p. 77). Les souris obtenues synthétisent une forte concentration d'hémoglobine humaine $\alpha 2-\beta A S 2$. Cette hémoglobine est effectivement très efficace pour inhiber la polymérisation de l'HbS dans des tests in vitro[2]. Naturellement, l'application éventuelle d'une telle stratégie aux drépanocytoses humaines nécessiterait que l'on sût transférer un transgène thérapeutique dans les cellules souches hématopoïétiques, ce qui reste, à ce jour, une difficulté non résolue. Il n'empêche que cet exemple illustre bien l'une des possibilités de thérapie génique des maladies dominantes.

[1. Hélène C, Saison-Behmoaras E. médecine/sciences $1994 ; 10$ : 253-73.]

[2. Mc Cune SL, et al. Proc Natl Acad Sci USA 1994 ; 91 : 9852-6.]

Découverte d'un nouvel inhibiteur de la croissance des neurones. Il existe des facteurs comme les neurotrophines et les molécules d'adhérence cellulaires qui favorisent la croissance et la régénération des cellules nerveuses [1]. Cependant, on sait que l'incapacité des cellules nerveuses du système nerveux central (SNC) chez l'adulte à se régénérer à la suite d'une lésion est due à la présence de molécules dans cet environnement qui inhibent la croissance des axones. Dans le numéro d'octobre de la revue Neuron, Lisa McKerracher et ses collègues de l'Université McGill rapportent que plusieurs protéines associées à la myéline (la matière blanche du cerveau) possèdent une des neurones [2]. Ces chercheurs ont fractionné des extraits de la myéline par un procédé de chromatographie. Les fractions purifiées furent ensuite testées sur des cellules de neuroblastomes en culture pour détecter la présence de molécules inhibitrices de la croissance des neurites. Cette approche a permis d'identifier la glycoprotéine associée à la myéline comme étant la source la plus importante d'inhibition de la croissance des neurites. Cette découverte pourrait avoir un impact important sur les recherches visant à régénérer les neurones du SNC à la suite de lésions.

[1. Schwab et al. Annu Rev Neurosi 1993 ; $16: 565-95$.]

[2. McKerracher L, et al. Neuron $1994 ; 13: 805-11$.]

L'ataxie épisodique est une maladie familiale rare, provoquant des crises d'ataxie généralisée sans manif estation entre les crises. Certaines formes s'accompagnent de myokymies (fasciculations musculaires). Des études de liaison ont localisé un gène d'une forme dominante dans une région où se trouve un ensemble de gènes de canaux $\mathrm{K}^{+}$. Des observations de recombinaisons ont abouti à la désignation d'un gène candidat probable sur le chromosome 12p, le gène KCNAI. On connaît la séquence codante de KCNA1 qui compte 1488 pb [1]. Un groupe de Portland (OR, USA) [2] a découvert dans quatre familles quatre mutations, toutes différentes, toutes des faux-sens, et à des positions conservées au cours de l'évolution. Trois d'entre elles sont dans une zone transmembranaire, la quatrième est dans une boucle intracytoplasmique. L'étude des mécanismes de cette maladie devra attendre les analyses, prévues, des effets des mutations par expression dans des ovocytes de xénope.

[1. Ramaswami M, et al. Mol Cell Neurosci 1990 ; 1 : 214-23.]

[2. Browne DL, et al. Nature Genet $1994 ; 8: 136-40$.]
L'immunité systémique intravaginale : un espoir pour l'induction d'une protection locale anti-VIH ? Les anticorps des muqueuses appartiennent habituellement au système immunitaire sécrétoire et sont indépendants du système immunitaire "systémique ". Ils inhibent la pénétration des agents pathogènes par différents mécanismes: agglutination, adhérence au mucus et masquage des adhésines [1]. Jusqu'à présent, on considérait que la protection immune de la muqueuse vaginale était assurée uniquement par des $\operatorname{IgA}$ sécrétoires et ne pouvait donc être induite que par voie muqueuse, mais cette induction est difficile à obtenir. En contestant le caractère uniquement sécrétoire de cette immunité locale et la nécessité d'une voie muqueuse d'immunisation, une équipe de l'Institut Pasteur vient de démontrer qu'une immunité cesvico-vaginale pouvait être facilement obtenue par injection parentérale [2]. En effet, sur des volontaires ayant subi un rappel de vaccination antitétanique, une réponse spécifique très élevée, de type systémique ( $\operatorname{IgG})$, a été observée simultanément dans le sérum et le fluide vaginal; l'avidité et l'activité spécifique (IgG anticorps/IgG totales) étaient similaires dans les deux milieux. Par ailleurs, l'efficacité antivirale d'anticorps $\operatorname{IgC}$ a été démontrée par protection passive intravaginale chez l'animal [3]. Dans l'optique d'une prévention de la transmission hétérosexuelle du SIDA, une vaccination parentérale pourrait donc induire des anticorps vaginaux potentiellement capables d'inhiber la pénétration du VIH et cela même en l'absence d'effet neutralisant in vitro - et de limiter ainsi son accès au système immunitaire.

[1. Iscaki I, et al. Bull Inst Pasteur 1994 ; 91 : 203-24.]

[2. Bouvet JP, et al. Infect Immun $1994 ; 62$ : 3957-61.]

[3. Whaley KJ, et al. I Infect IDis 1994; $169: 647-9$. 
- Absence d'empreinte génomique sur le chromosome 7 masculin. Nous avons récemment relaté dans médecine/sciences $\left(n^{\circ} 11\right.$, vol. 10$)$, $p .1169)$ que quatre sujets présentant une isodisomie du chromosome 7 d'origine maternelle, totale ou au moins du bras long, étaient de taille réduite. On peut donc envisager l'existence d'une "empreinte génomique " sur ce chromosome. Un groupe finlandais [1] vient de rapporter un cas d'isodisomie 7 chez une femme de 23 ans de taille normale, isodisomie dont l'origine est paternelle. Ce diagnostic a pu être porté parce que le sujet était porteur d'une diarrhée congénitale au chlore, dont le gène a été récemment assigné au chromosome 7 [2]. Cette observation prouve qu'il n'y a pas d'effet d'empreinte lorsque la disomie est paternelle.

[1. Höglund P, et al. Am J Hum (ienet $1994 ; 55: 747-52$.

[2. Kere J, et al. Proc Natl Acad Sici USA 1993; 90 : 10686-9.]
La mort cellulaire dans le thymus enfin démontrée. I e répertoire des lymphocytes $T$ est sélectionné dans le thymus selon deux critères. Une sélection positive sauve de la mort cellulaire programmée les thymocytes ayant une affinité appropriée pour les molécules du complexe majeur d'histocompatibilité $(\mathrm{CMH})$. Une sélection négative élimine activement les thymocytes potentiellement autoréactifs. Un faible pourcentage de thymocytes immatures survit à cette double sélection alors que la vaste majorité d'entre eux meurt par apoptose. I.es techniques classiques d'histochimie n'ont pourtant pas pu montrer que le thymus est le siège d'une mort cellulaire massive. Il a fallu attendre de pouvoir détecter les cassures d'ADN in situ pour mettre en évidence l'apoptose dans le thymus. Sur coupes de thymus, on peut maintenant voir les cellules apoptotiques dispersées dans le cortex et localement englouties dans des ma- crophages. En étudiant les souris mutantes n'exprimant pas le $\mathrm{CMH}$, les auteurs montrent que les cellules apoptotiques dans le cortex sont probablement les cellules qui n'ont pas été sélectionnées positivement. En croisant judicieusement certaines lignées de souris, ils démontrent que les thymocytes négativement sélectionnés meurent dans la medulla. I.es macrophages qui débarrassent le thymus des cellules apoptotiques ont des phénotypes différents selon qu'ils agissent dans le cortex ou la medulla. Si l'on considère l'ampleur du phénomène d'apoptose dans le thymus, la part due à la sélection négative semble être minime et la grande majorité des cellules meurent parce qu'elles n'ont pas été sélectionnées positivement.

[Surh CD. Nature $1994 ; 372: 100$ 3.] 